\title{
HE_OWW Filter for Enhancement of Wildlife Observation Digital Images in Limited Light Condition
}

\author{
S. Suhaila, S. Lee, R. Hazli, N.S.A.M. Taujuddin\& N.S. Suriani \\ Universiti Tun Hussein Onn Malaysia, Malaysia, suhailas@uthm.edu.my
}

\begin{abstract}
Image enhancement provides human with better visual quality effects. However, images that are captured in low light condition are usually low-visibility and degraded by different noise types such as Poisson noise. Thus, this study goal is to develop an effective enhancement technique for low light condition wildlife observation digital image. This study proposed an effective enhancement technique which combines contrast enhancement technique and denoising technique. The proposed technique, HE_OWW filter utilizes Histogram Equalization and OTSU WIE-WATH filter to increase the visibility of low light image and remove Poisson noise found in wildlife observation image due to low light intensities. The performance of proposed method is evaluated through objective and subjective measure. For objective measure, the proposed method is analyzed by Root Mean Square (RMS) Contrast, luminance, Perceptual Sharpness Index (PSI) and computational time. Meanwhile, subjective measure is done by visual effects inspection. Proposed method provided the best result in limited light source image with RMS Contrast of 0.6004, luminance of 0.4354 and PSI of 0.4492 and took the shortest time to compute the test images. The result shows that proposed method is the best among compared techniques for enhancement of wildlife observation image with low light source.
\end{abstract}

Key words : Digital Images, Histogram Equalization, Retinex, Wavelet, Threshold

\section{INTRODUCTION}

Generally, image enhancement techniques are used to improve the interpretability or highlight certain details in the image so that the viewers can get a better visual effect and provide better input for the image denoising process [1]. Principal objective of image enhancement is to modify one or more of the attributes of digital image and make it applicable in different areas of science and engineering. In addition to different light level conditions, quality of an image is also affected by environmental disturbances and external noises.
Noise is unwanted effect that is produced in an image and could be seen as grains [2]. Differ from day-time image acquisition, images taken in limited light condition usually suffer from noise and motion blur. Different types of noise will degrade the quality of digital image in different ways. Noises that commonly found in an image are Gaussian noise, Poisson noise, Salt and Pepper Noise, and others [3].

Images captured at night usually seemed dark due to low light intensity. Limited light condition means that the surrounding area where the image is to be captured has no light or weak light source, such as taking photo at night or in a dark room [3]. Taking a superior quality of picture in limited light condition is always harder than a good light condition. It would be a large challenge for a non-professional photographer to take a limited light condition digital image.

In this high-tech era, digital cameras are compatible with a different mode of camera setting that is used for capturing image in different condition. However, there is still a big challenge for a photographer to get a good quality of wildlife observation digital images. A flash light is required to take a picture in limited light condition. However, flash light is not applicable for capturing wildlife image in dark scene, because the strong emission of flash light could frighten the animals, and it is only effective for nearby object [4]. Therefore, long exposure time setting is required to avoid the visible flash. However, long exposure time is not suitable for capturing limited light wildlife observation digital image, due to scene motion or camera shake [5]. Colour image that are captured using short exposure time is not blurry, but contain lots of noise due to high ISO. The ISO in digital image is referred to the measure of sensitivity of image sensor [6]. High ISO is generally used in dark condition, but it will amplify both image signal and noise in image.

Although there are lots of existing image denoising and image enhancement techniques, but only few of them are specified for limited light condition digital image, especially focused on real application such as wildlife observation. Common problems found in denoising techniques are long computational time and loss of image fine scale details such as over smoothing of texture structures [7]. Apart from that, existing image enhancement techniques suffers from degraded edges after the enhancement process [8]. 
Therefore, this study proposed an effective image enhancement technique for limited light condition wildlife observation digital image. Image enhancement technique is collaborated with image denoising technique to modify the attributes of noisy image and removal of Poisson noise in a short computational time. The proposed technique, HE_OWW filter, combines the Histogram Equalization and the OTSU WIE-WATH filter and it would be useful to enhance digital image that is taken in limited light condition.

\section{LITERATURE REVIEW}

In the past few years, several techniques have been discovered for image enhancement and noise removal. This section discusses the drawbacks and advantages of the existing image enhancement and denoising methods.

\subsection{Existing Image Enhancement Methods}

The findings of Multi-Scale Retinex computation was published in an article named "Multi-Scale Retinex for Colour Image Enhancement" [9]. The proposed algorithm could overcome the limitation of Single Scale Retinex by combining several Single Scale Retinex output image. The proposed method assembled the dynamic range compression of the small scale retinex with the tonal rendition of the large scale retinex so that the output image encompasses both. This technique produced excellent dynamic range compression, but poor in tonal rendition.

Furthermore, Histogram Equalization for color image enhancement was introduced in the article of "Hue-Preserving Color Image Enhancement without Gamut Problem" [10] to improve the dynamic range of a low contrast image. Initially, the RGB image is converted into HSV format which has three separate channels of Hue, Saturation and Value. Then, Histogram Equalization technique is applied to Value channel independently without affecting the other two channels. After the Histogram Equalization process, three-colour channels are combined again to form an enhanced HSV image. Then, this enhanced HSV image is converted back to RGB format to get the enhanced output image. This technique is simple and efficient to increase the contrast of image, but it may produce unnatural effect in the images.

According to research in [11], the proposed method firstly inverted the input of low-light video. After the inverting process, the pixels in the sky of the inverted videos have high intensities in all colour (RGB) channels but non-sky region suffer from low intensities in one of the colour channel. Hence, the author applied an optimized image de-haze algorithm on inverted video. De-haze is applied to perform haze removal on the inverted video frames to get a better output. However, the inverted low-light image looked like hazy image and it seemed unrealistic.
In the year 2017, an improved Adaptive Histogram Equalization [12] is introduced by transforming each pixel with a transformation function derived a neighbourhood area. Each pixel is transformed based on the histogram of a square encompassing the pixel. The derivation of the transformation functions from the histograms is precisely the same as for customary histogram equalization. The transformation function is corresponding to the cumulative distribution function $(\mathrm{CDF})$ of pixel values in the area. The proposed method had successfully improved the visibility of images with lowest MSE and highest PSNR value. However, it could not adopt with varying distances of captured images.

\subsection{Existing Image Denoising Methods}

Bilateral filtering [13] has been proposed for grayscale and colour images based on their geometric location and photometric similarity. Near values are preferred to distant values in range and domain. Bilateral filtering produced no phantom colours along edges in colour images, and reduced phantom colours where they appeared in the original image. The method is edge preserving, non-iterative, local, and simple. However, it will has intensity plateaus which causes the images to be cartoon-likes and introduces false edges in the image.

According to research in [14], a multi-stage noise removal method was developed by using the Bilateral filter and wavelet thresholding. Wavelet transform based energy is used for measuring the content in each image frame and selecting a suitable reference frame so that other frames can be aligned with respect to the reference frame. In the first stage of noise removal, the Bilateral filter is used for refining the edge preservation. In the second stage, discrete wavelet transform is used to decompose the image to remove noises in uniform regions and edges. However, this method is only applicable for mobile implementation.

There is another denoising technique presented in 2013, which is developed from the hybridization of the Bilateral filter and the Wavelet Thresholding [15] to remove Poisson noise of digital image in low light condition. The noisy image is passed through the Bilateral filter in the earlier stage to remove noise but caused the images to be blurred. Hence, output image from the Bilateral filter is passed through wavelet threshold to reduce the blurring part and refine the details in the image. The proposed method is evaluated using the Peak Signal to Noise Ratio (PSNR), Mean Squared Error (MSE) and visual effects. These three evaluation methods had proved that the hybridization method is a better way to preserve the edge while eliminating Poison noise in digital image. Nevertheless, the hybridization method is not effective in removing Salt and Pepper noise.

In the year 2018, the OTSU WIE-WATH filter is presented by Y. Y. Chia to remove Poisson noise in low light condition [16]. These denoising technique is developed based on the 
Otsu Threshold, Wiener filter and Wavelet Threshold. The OTSU WIE-WATH filter is intended to remove low and medium level of Poisson noise. Experimental result shows that the proposed filters could remove different level of Poisson noise effectively, but the computational time of proposed filter is slightly longer than other conventional filters.

\section{METHODOLOGY}

This section includes workflow and methodology of the study. The study is developed by using MathWork of Matlab software. The techniques of image processing used in this study could be divided into two main parts, which are image enhancement followed by image denoising. In the first part, a contrast enhancement technique named Histogram Equalization [10] is utilized to enhance limited-light images. Meanwhile, in the second part of the study, the OTSU WIE-WATH filter [16] is used to remove the Poisson noise. Furthermore, an image enhancement technique, HE_OWW filter, which combines the Histogram Equalization and the OTSU WIE-WATH filter is proposed in this study to enhance the limited light condition wildlife observation image effectively.

All the techniques mentioned above will be tested with data set of limited-light condition of wildlife observation digital images. The effectiveness of these techniques will be determined and analysed in this study as well.

\subsection{Proposed Method: HE_OWW Filter}

Proposed method, HE_OWW filter, is developed by combining the Histogram Equalization [10] and the OTSU WIE WATH filter [16]. This method is proposed for enhancement of wildlife observation digital images in limited-light condition.

Histogram Equalization is able to increase the contrast of limited light image whereas the OTSU WIE-WATH filter is effective for Poisson noise removal. Histogram equalization is used to adjust image intensities to enhance contrast by increases the global contrast of the image. Thus, the pixel intensities can be better distributed on the histogram, which allows for areas of lower local contrast to gain a higher contrast. On the other hand, the WIE-WATH Filter utilizes the Wavelet Threshold and the Wiener Filter [17]. This method is proposed because the WIE-WATH Filter has been proved effective in removing Gaussian noise [17] and Poisson noise [16]. The Otsu Threshold is beneficial in classifying the image into smooth and texture regions. The OTSU WIE-WATH Filter only denoises the smooth regions of noisy images using the combination of the Wiener filter and the Wavelet Threshold (WIE-WATH filter) and the texture regions remain the same as the original noisy image pixels. The proposed filter allows the noisy pixels in the dark regions to be removed while preserving the texture regions to avoid over smoothing of test images [16].

Therefore, the proposed method utilizes the advantages of both the Histogram Equalization to enhance image contrast and the OTSU WIE-WATH filter in removing noise while preserving details to enhance the limited light condition wildlife observation image.

\subsection{Histogram Equalization}

The Histogram Equalization [10] is one of the contrast enhancement techniques that enhanced the image by using its histogram. This technique is used to increase the global contrast of the image and distribute the intensity of image in dynamic range with a flatten histogram while preserving hue and saturation component. However, the Histogram Equalization was then modified so that it is applicable for the RGB image.

First of all, an RGB image, $x(i, j)$ is inserted and it is then converted into the HSV format, h(i,j) that consists of Hue $(\mathrm{H})$, Saturation (S), and Value (V) channel. Histogram Equalization is applied onto $\mathrm{V}$ channel without affecting $\mathrm{H}$ and $\mathrm{S}$ channel. After the application of Histogram Equalization, $\mathrm{H}, \mathrm{S}$ and $\mathrm{V}$ channels are resembled again to form a new HSV image, $s(i, j)$. Lastly, the HSV image, $s(i, j)$ is converted back to the RGB format to formed the output image, $r(\mathrm{i}, \mathrm{j})$.

The computation for the Histogram Equalization, $\mathrm{T}(\mathrm{x})$, is represented by:

$x^{\prime}=T(x)=\sum_{s=0}^{x} n_{s} \cdot \frac{\text { max. intensity }}{N}$

where,

$n_{s}$ : number of pixels at intensity $s$,

$N$ : total number of pixel in the image $x$

\subsection{OTSU WIE-WATH Filter}

The OTSU WIE-WATH filter [16] was proposed by Chia Yun Yi in 2018. It employs hybridization of several denoising technique to reduce the Poisson noise effectively. This technique is made up of three denoising methods, which are the Otsu Threshold, Wavelet Threshold and Wiener filter. This method is able to remove the noise in smooth region whereas information in texture region remain unchanged.

First of all, noisy limited light condition wildlife observation digital image, $r(i, j)$ will be processed with the Otsu Threshold to generate an Otsu binary image, obw $(i, j)$. The Otsu binary image consist of level 1 and level 0 . Level 1 denotes texture regions whereas level 0 denotes smooth region. 
The WIE-WATH Filter utilizes Wavelet Threshold and Wiener Filter [17]. At first, the Discrete Wavelet Transform (DWT) transforms noisy image into sub-bands. After calculating the variance, the Wiener filter is apply on each sub-band by using local window $\mathrm{n} \times \mathrm{n}$. Finally, the inverse DWT (IDWT) is performs to obtain the denoised image, $w(i, j)$.
In smooth region, the WIE-WATH filter is applied to the location of level 0 in Otsu binary image. Meanwhile, the pixel value on texture region will remain unchanged. Finally, the OTSU WIE-WATH image, ow(i,j) is formed by combining noisy image and the WIE-WATH filtered image. The flow chart of the algorithm is shown in Figure 1.

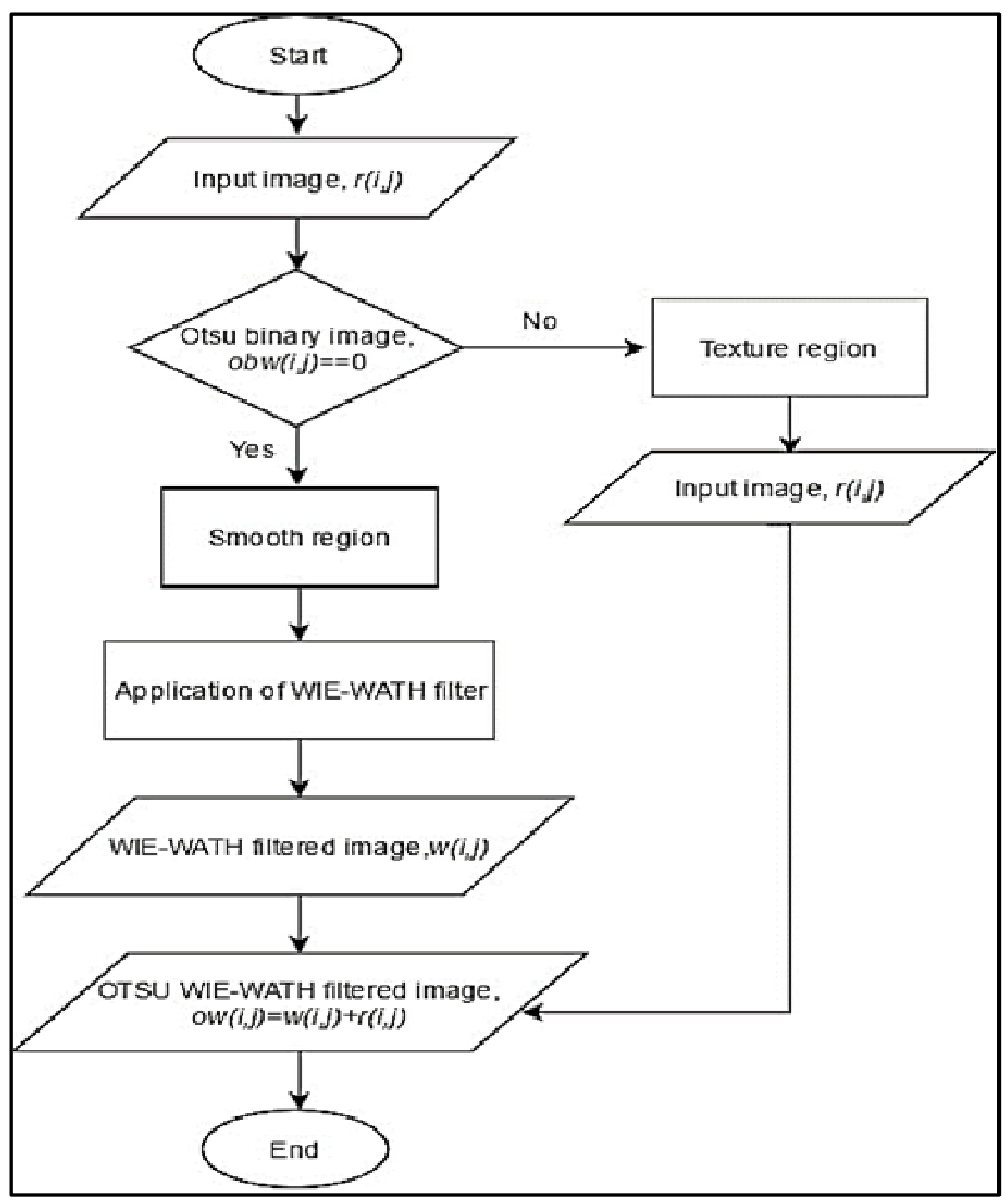

Figure 1: Flowchart for OTSU WIE-WATH filter

\subsection{Performance Analysis}

Performance of denoising and enhancement method is measured by objective evaluation in terms of the RMS contrast, luminance, PSI and computational time. The subjective evaluation method used for evaluation is visual effects inspection.

The common way to define contrast of a single image is the Root Mean Square (RMS) Contrast [17], which calculate the contrast based on the standard deviation of luminance levels in the stimulus.

The definition of RMS Contrast is given by:

$$
\text { RMSContrast }=\left[\frac{1}{M N} \sum_{i=0}^{N-1} \sum_{j=0}^{M-1}(y[i, j]-\tilde{y})^{2}\right]^{\frac{1}{2}}
$$

where,

$y(i, j)$ : intensities of the output enhanced image, $y(i, j)$ of size $\mathrm{M}$ and $\mathrm{N}$

$\tilde{y} \quad$ : average intensity of all pixel in the output image, $y(i, j)$

The intensity is normalized to between the ranges of 0 to 1 . Higher value of the RMS Contrast indicates better performance of output enhanced image.

The luminance [18] can be extracted in the $\mathrm{Y}$ channel during the conversion of the RGB to YIQ. Luminance is the parameter used to measure the overall brightness or darkness of an image.

The computation of Luminance is given by:

Luminance $=0.299 \mathrm{R}+0.587 \mathrm{G}+0.114 \mathrm{~B}$ 
where $\mathrm{R}, \mathrm{G}$ and $\mathrm{B}$ represents the red, green and blue components in output enhanced image, $y(i, j)$ respectively. Higher value of luminance indicates better performance of enhanced image.

The Perceptual Sharpness Index, PSI, [19] is a no-reference image sharpness estimation method based on local edge gradient analysis. An image sharpness index can be used to measure the effectiveness of denoising algorithms [20]. The value of the PSI will be given in the range between 0 and 1 . The calculation of Perceptual Sharpness Index is given by:

$P S I=\left(I_{\max }(y)-I_{\min }(y)\right) / w(y)$

where,

$w(y)$ : edge width of output enhanced image, $y(i, j)$

$I_{\max }(y)$ : local maximum luminance pixels of output enhanced image, $y(i, j)$

I min $(y)$ : local minimum luminance pixels of output enhanced image, $y(i, j)$

The higher value of the PSI value denoted the better quality of output enhanced image.

Meanwhile, the visual effects inspection is performed based on observation using human eye-sight. Visible characteristics that can be observed in an image includes brightness, edges, fine details and noises.

\section{RESULTS AND DISCUSSION}

Development of image enhancement technique for limited light condition wildlife digital image can be divided into two parts: contrast enhancement, and noise removal. Contrast enhancement part could increase the contrast of image and thus recall information in dark regions. Meanwhile, noise removal part is necessary to remove Poisson noise that is being amplified during contrast enhancement process. In this study, an effective enhancement technique, HE_OWW filter, which combined the Histogram Equalization and the OTSU WIE-WATH filter is proposed for enhancement of images captured in limited light condition. The performance of proposed technique is evaluated in terms of the RMS contrast, luminance, PSI, computational time and visual effects inspection to determine the effectiveness of the algorithm.

\subsection{Performance of Proposed Method for Limited Light Source Image}

Table 1 shows the parameter settings for denoising techniques utilized for performance comparison.

Table 1: Parameter Setting For Enhancement Techniques

\begin{tabular}{|c|c|c|}
\hline \multirow{2}{*}{ Enhancement Techniques } & Parameters & Settings \\
\hline \multirow{4}{*}{ Proposed, HE_OWW } & Threshold value & {$[01]$} \\
\cline { 2 - 3 } & $p$ & 30 \\
\cline { 2 - 3 } & Wiener factor, $\tilde{n}$ & 0.001 \\
\cline { 2 - 3 } & Wiener mask size, $n$ & $3 \times 3$ \\
\hline HE[10]+BL[13] & DWT decomposition level & 2 \\
\hline \multirow{2}{*}{ HE[10]+BL[13]+WT[17] } & Window size, $w$ & 5 \\
\cline { 2 - 3 } & Dindow size, $w$ & 5 \\
\hline
\end{tabular}

Enhancement techniques that have been selected for comparison in this study includes hybridization of the Histogram Equalization and the OTSU WIE-WATH filter (proposed), HE_OWW filter, hybridization of the Histogram Equalization and the Bilateral filter, $\mathrm{HE}+\mathrm{BL}$, and hybridization of the Histogram Equalization, Bilateral filter and the Wavelet Threshold, HE + BL + WT.
The performance of enhancement techniques are analyzed using two types of evaluation method, which are objective evaluation and subjective evaluation. Objective evaluation is done by calculating the RMS contrast, luminance, Perceptual Sharpness Index (PSI) and computational time.

Table 2: Comparison of Enhancement Techniques for Objective Measure

\begin{tabular}{|c|c|c|c|c|}
\hline \multirow{2}{*}{$\begin{array}{c}\text { Enhancement } \\
\text { Techniques }\end{array}$} & \multicolumn{4}{|c|}{ (Evaluation Techniques) } \\
\cline { 2 - 5 } & RMS Contrast & Luminance & PSI & Computational Time(s) \\
\hline Proposed, HE_OWW & $\mathbf{0 . 6 6 6 0}$ & $\mathbf{0 . 3 6 7 3}$ & $\mathbf{0 . 6 3 5 2}$ & $\mathbf{3 . 5 7 8 1}$ \\
\hline HE+BL & 0.6494 & 0.3614 & 0.5487 & 42.2656 \\
\hline HE+BL+WT & 0.6263 & 0.3580 & 0.3848 & 44.0625 \\
\hline
\end{tabular}


Based on result in Table 2, proposed method, the HE_OWW filter that combined the Histogram Equalization and the OTSU WIE-WATH filter shows the highest average RMS Contrast for limited source test images. The RMS Contrast of proposed method is 0.6660 , which is 0.0397 higher than the $\mathrm{HE}+\mathrm{BL}+\mathrm{WT}$ filter.

In terms of luminance, the proposed method is the highest among other compared methods. The HE_OWW filter has the luminance of 0.3673 .

Furthermore, the proposed method, HE_OWW filter, also provides the highest PSI of 0.6352 . When compared to the $\mathrm{HE}+\mathrm{BL}$ filter, proposed method gives an increment of 0.0865 .

Results obtained in term of computational time shows that the HE_OWW filter took the shortest time to compute an image. The computational time for the HE_OWW filter is 3.5781 seconds, which is much faster than the HE+BL filter and the $\mathrm{HE}+\mathrm{BL}+\mathrm{WT}$ filter. There is a decrement of 38.6875 seconds when compared to the $\mathrm{HE}+\mathrm{BL}$ filter. When compared with computational time of the $\mathrm{HE}+\mathrm{BL}+\mathrm{WT}$ filter, the proposed method gives a decrement of 40.4844 seconds.

Figure 2 and Figure 3 show the limited light source test images that are enhanced by the proposed method, HE_OWW filter, HE+BL filter, and HE+BL+WT filter. It can be observed that all techniques are able to increase the contrast and brightness of the image for better visual effects. The proposed method, HE_OWW filter, which combined the Histogram Equalization and the OTSU WIE-WATH filter removed most of the noise in the test images with the preservation of details in the texture region. Meanwhile, the test image that is enhanced by other techniques shows blurred effect in the texture region.

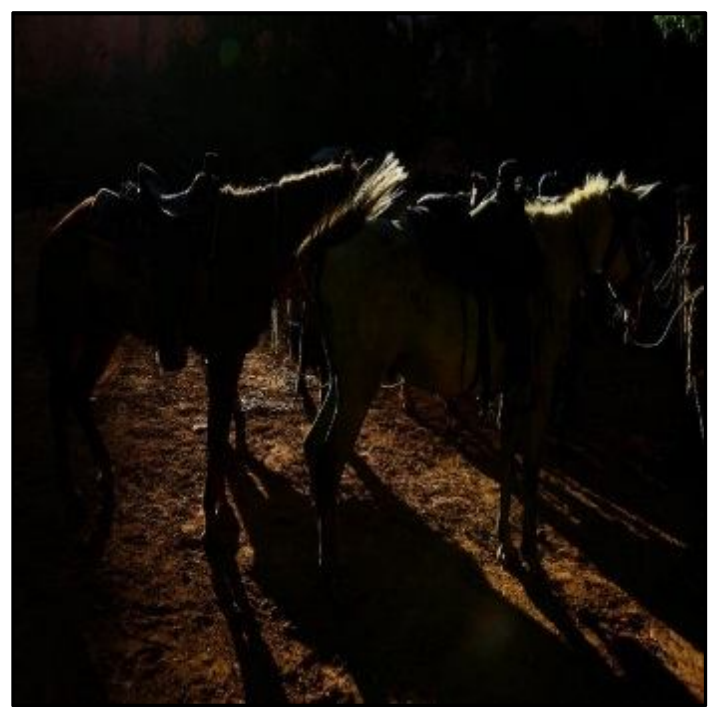

(a) Original Image

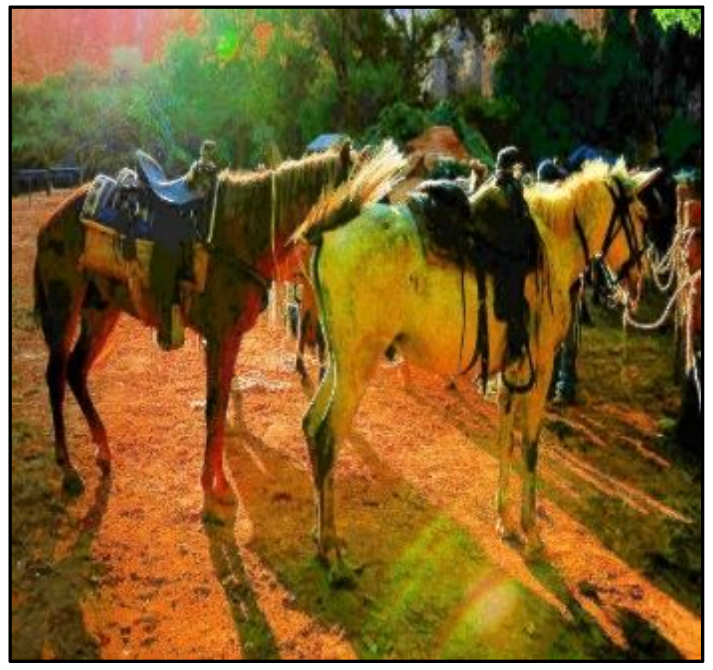

(b) Proposed HE_OWW Filter

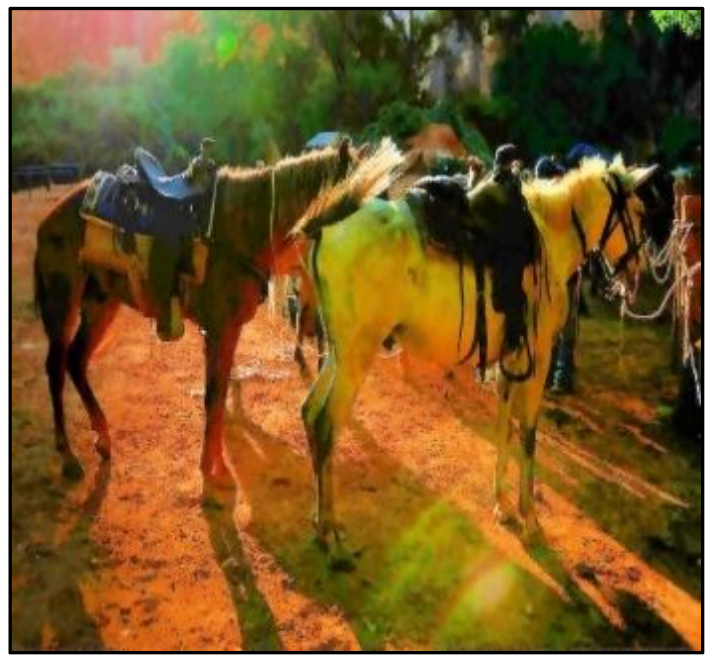

(c) $\mathrm{HE}+\mathrm{BL}$

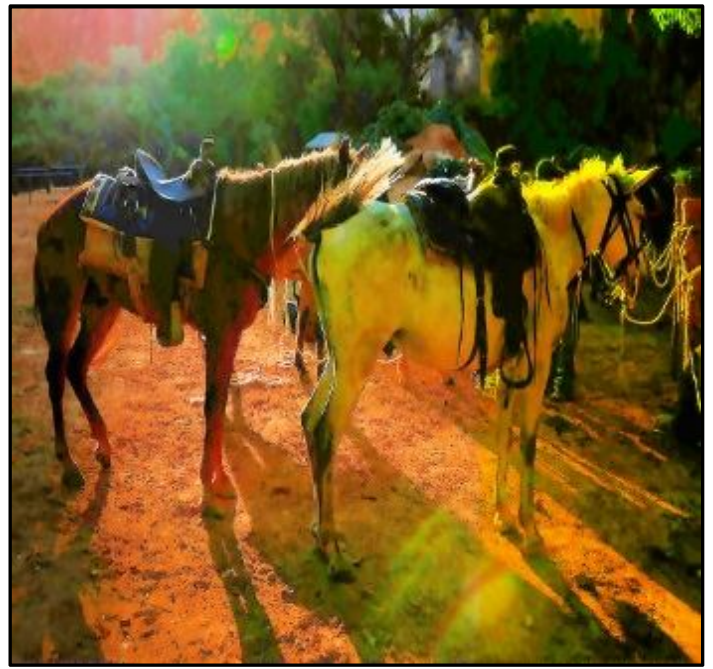

(d) $\mathrm{HE}+\mathrm{BL}+\mathrm{WT}$

Figure 2: Visual effects for enhanced limited light source of "Horse" [21] image. (a) Original limited light source image. [21,22]

(b) to (e) respectively denote the results using different enhancement methods as proposed method; $\mathrm{HE}+\mathrm{OWW}, \mathrm{HE}+\mathrm{BL}$ and $\mathrm{HE}+\mathrm{BL}+\mathrm{WT}$ 


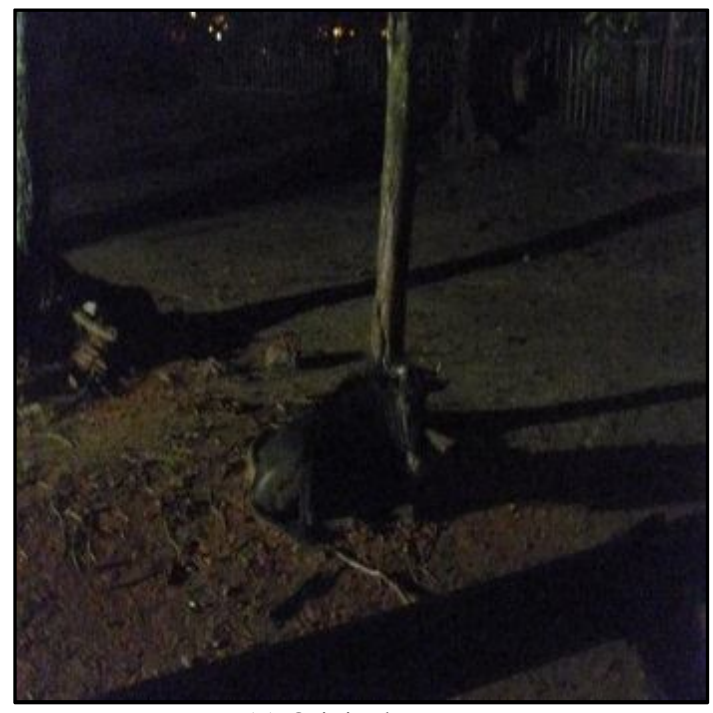

(a) Original Image

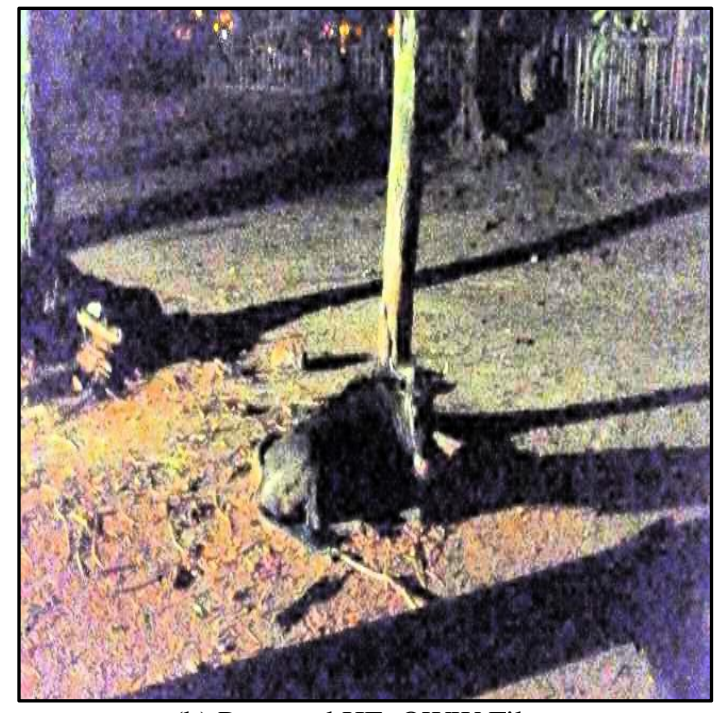

(b) Proposed HE_OWW Filter

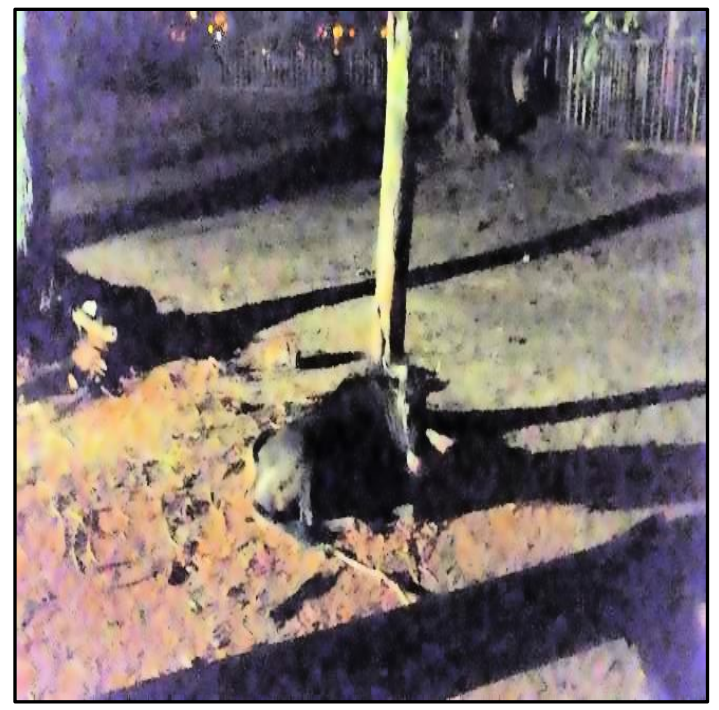

(c) $\mathrm{HE}+\mathrm{BL}$

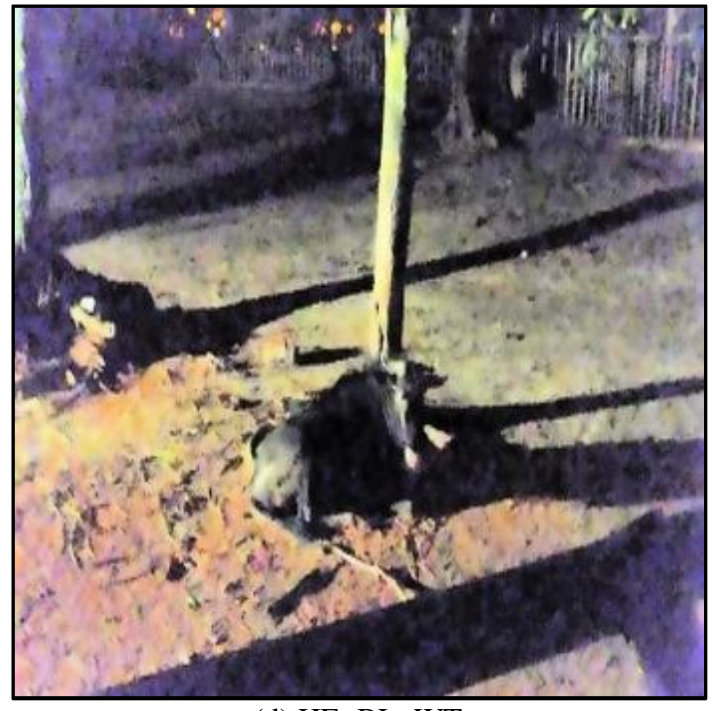

(d) HE+BL+WT

Figure 3: Visual effects for enhanced limited light source of "Goat"(right) image. (a) Original limited light source image. [21] (b) to (e) respectively denote the results using different enhancement methods as proposed method; $\mathrm{HE}+\mathrm{OWW}, \mathrm{HE}+\mathrm{BL}$ and $\mathrm{HE}+\mathrm{BL}+\mathrm{WT}$

\section{CONCLUSION}

Image enhancement is a challenging issue especially in limited light condition. Noise is often found in limited light condition digital image, and caused the degradation of image quality. In this study, an effective enhancement method, HE_OWW filter, which combines the Histogram Equalization and the OTSU WIE-WATH filter is proposed to give the best solution for the limited light condition wildlife observation digital image. Proposed method, HE_OWW filter, outperformed other compared methods in all aspects, including the RMS Contrast, luminance, PSI, computational time and visual effect inspection. Proposed method provided the best result in limited light source image with the RMS Contrast of 0.6004 , luminance of 0.4354 and the PSI of 0.4492 and took the shortest time to compute the test images. The proposed method is able to increase the visibility of the wildlife observation limited light condition image with details preservation.

\section{ACKNOWLEDGEMENT}

The authors would like to express our gratitude for the support given by Universiti Tun Hussein Onn Malaysia throughout the duration of this study.

\section{REFERENCES}

1. S. Mahajan, and R. Dogra, "A review on image enhancement techniques," International Journal of Engineering and Innovative Technology, vol. 4, no. 11, pp.108-113, 2015.

2. L. Li, R. Wang, W. Wang, and W Gao, "A low-light image enhancement method for both denoising and 
contrast enlarging," Proceedings of IEEE International Conference on Image Processing, pp. 3730-3734, 2015.

3. V. Rohit, and J. Ali, "A comparative study of various types of image noise and efficient noise removal techniques," International Journal of Advanced Research in Computer Science and Software Engineering, vol. 3, no. 10, pp. 617-622, 2013.

4. K. Takeuchi, M. Tanaka, and M. Okutomi, "Low-light scene color imaging based on luminance estimation from near-infrared flash image," Proceedings of IEEE International Workshop on Computational Cameras and Displays, 2013.

5. S. Matsui, T. Okabe, M. Shimano, and Y. Sato, "Image enhancement of low-light scenes with near-infrared flash images," Proceedings of Asian Conference on Computer Vision, pp. 213-223, 2010. https://doi.org/10.1007/978-3-642-12307-8_20

6. X. Li, "Blind restoration of very-high-ISO photos via low-rank methods," Proceedings of IEEE International Conference on Multimedia and Expo.pp.1-6, 2014.

7. W. Zuo, L. Zhang, C. Song, and D. Zhang, "Texture enhanced image denoising via gradient histogram preservation," Proceedings of IEEE Conference on Computer Vision and Pattern Recognition. pp.1203-1210, 2013. https://doi.org/10.1109/CVPR.2013.159

8. R. C. Gonzalez, and R. E. Woods, "Digital image processing," 3rd Ed. United States: Prentice Hall. 2007.

9. Z. Rahman, D. J. Jobson, and G. A. Woodell, "Multi-scale Retinex for color image enhancement," Proceedings of IEEE International Conference on Image Processing, vol. 3, pp.1003-1006, 1996.

10. S. Naik, and C. Murthy, "Hue-preserving color image enhancement without Gamut problem," IEEE Transactions on Image Processing, vol. 12, no. 12, pp.1591-1598, 2003. https://doi.org/10.1109/TIP.2003.819231

11. X. Dong, G. Wang, Y. Pang, W. Li, J. Wen, W. Meng, and Y. Lu, "Fast efficient algorithm for enhancement of low lighting video," Proceedings of IEEE International Conference on Multimedia and Expo.pp. 1-6, 2011.

12. G. S. Jatana, and Pooja. "adaptive histogram equalization technique for enhancement of coloured image quality," International Journal of Latest Trends in Engineering and Technology, vol. 8, no. 2, pp.10-17. 2017. https://doi.org/10.21172/1.82.002

13. N. H. Kaplan, I. Erer, and N. Gulmus, "Remote sensing image enhancement via Bilateral filtering," Proceedings of IEEE International Conference on Recent Advances in Space Technologies (RAST), pp.139-142, 2017. https://doi.org/10.1109/RAST.2017.8002981

14. R. P. M. Kini, S. Khunteta, S. Garg, K. Yeturu, and S. Kar, "Multi-frame low illumination noise removal in smartphone," Proceedings of Annual IEEE India Conference, pp. 1-5, 2013.

15. S. Suhaila, S. Z. H. Al Fakkri, R. Hazli, and Z. Tukiran, "development of denoising method for digital image in low-light condition," Proceeding of IEEE International Conference on Control System, Computing and Engineering, pp. 142-147, 2013.
16. S. Suhaila, Y. Y. Chia, R. Ibrahim, R. Hazli, and N. S. A. M. Taujuddin, "New wavelet domain Wiener filter based denoising for Poisson noise removal in low-light condition digital image (OTSU WIE-WATH)," Journal of Telecommunication, Electronic and Computer Engineering (JTEC), vol. 10, no.1-4, pp.107-114, 2018.

17. M. M. Siddeq, "Using discrete Wavelet transform and Wiener filter for image de-noising," Journal for Science and Medicine, vol. 2, pp.18-30, 2009.

18. K. Lakhwani, P. D. Murarka, and N. S. Chauhan, "Color space transformation for visual enhancement of noisy color image," International Journal of ICT and Management, vol. 3, no. 2, pp. 9-13, 2015.

19. C. Feichtenhofer, H. Fassold, and P. Schallauer, "A perceptual image sharpness metric based on local edge gradient analysis," IEEE Signal Processing Letters, vol. 20, no.4, pp. 379-382, 2013. https://doi.org/10.1109/LSP.2013.2248711

20. S. Jiao, H. Qi, and W. Lin, "No-reference perceptual image sharpness index using normalized DCT-based representation," Proceedings of IEEE International Symposium on Computational Intelligence and Design, pp.150-153, 2014. https://doi.org/10.1109/ISCID.2014.50

21. "Horses," $2013 . \quad$ [Online]. Available: https://www.flickr.com/photos/ 100915707@N02/96152 18617/in/ photostream/.

22. Jennifer E. Sabugaa, Glenn S. Lahayon. Digital Data Classification and Extraction for Records Management of PAPS and PACS Documents. International Journal of Advanced Trends in Computer Science and Engineering. Volume 9, No.1.1, 2020 https://doi.org/10.30534/ijatcse/2020/4891.12020 